\title{
Immunological tolerance
}

\author{
Harald von Boehmer ${ }^{1,2 *}$ and Herman Waldmann ${ }^{3 *}$ \\ 1 Lab of Lymphocyte Biology, Department of Cancer Immunology and AIDS, Dana Farber Cancer Institute, Boston, MA, USA \\ 2 Harvard Faculty of Arts and Sciences, Harvard University, Cambridge, MA, USA \\ 3 Sir William Dunn School of Pathology, University of Oxford, Oxford, England \\ *Correspondence: harald_von_boehmer@dfci.harvard.edu or herman.waldmann@path.ox.ac.uk
}

\section{GRAND CHALLENGES}

The question of self-non-self discrimination, and thereby immunological tolerance, is as old as our appreciation of the receptor-diversity in the immune system. Initially negative selection of self-reactive lymphocytes by clonal deletion in primary lymphoid organs, enhanced by promiscuous expression of ectopic antigens in these organs, attracted attention. It became clear, however, that such central deletional mechanisms were some of many used to achieve non-responsiveness. Additional "recessive" mechanisms such as deletion and/or anergy in secondary lymphoid organs were also discovered, but it became clear that even these additional passive mechanisms were insufficient to account for self-tolerance and that "dominant" immunoregulation played a major, indeed essential, role. The discovery of the transcription factor Foxp3, as the master switch enabling regulation, has led to the identification of Foxp3 expressing cells whose sole role is suppression of the functions of other adaptive and innate immune cells.

These facts make it necessary to study the mechanisms of immunological tolerance at the cellular and molecular level in general, and the development, activation and effector function of regulatory Tcells in particular, with the same intensity as has been applied to investigating immunity to foreign antigens. Once the fine details of tolerance are understood we should be in a better position to study how tolerance fails in autoimmune diseases, and to develop new ways of reprogramming the immune system so as to amplify tolerance mecha- nisms in autoimmunity, transplantation and allergy, and to override them to boost immunity to cancer. Thus the goals for the future are the understanding of self-nonself discrimination at the mechanistic level, as well as application of the knowledge to achieve a better understanding of human disease and its rational treatments.

Received: 29 October 2010; accepted: 01 November 2010; published online: 22 November 2010.

Citation: von Boehmer $H$ and Waldmann $H$ (2010) Immunological tolerance. Front. Immun. 1:102. doi: 10.3389/fimmu.2010.00102

This article was submitted to Frontiers in Immunological Tolerance, a specialty of Frontiers in Immunology.

Copyright $\odot 2010$ von Boehmer and Waldmann. This is an open-access article subject to an exclusive license agreement between the authors and the Frontiers Research Foundation, which permits unrestricted use, distribution, and reproduction in any medium, provided the original authors and source are credited. 